\title{
Semi-Automatic 3D-Segmentation of Live-3D Echocardiographic Images
}

\author{
T Boettger ${ }^{1}$, I Wolf ${ }^{1}$, T. Kunert ${ }^{1}$, S Mottl-Link ${ }^{2}$, M Hastenteufel ${ }^{1}$, \\ R De Simone ${ }^{2}$, HP Meinzer ${ }^{1}$ \\ ${ }^{1}$ German Cancer Research Center, Heidelberg, Germany \\ ${ }^{2}$ Chirurgische Universitätsklinik, Heidelberg, Germany
}

\begin{abstract}
This paper presents a new segmentation tool for Live-3D echocardiographic images. It is based on a discrete three-dimensional deformable model. The model deformation scheme was extended by an interactive force component which allows to take advantage of the accuracy of user driven segmentation tools. Various interaction schemes were implemented to allow for interactive correction of the deformable model's development. By directly incorporating the user defined attractors into the deformable model, the deformation process is accellerated and model parametrization is simplified. The user can draw points and/or contours into the image data instead of adjusting model parameters. The new tool was successfully used for segmentation of both left $(L V)$ and right ventricles $(R V)$ from Live-3D echocardiographic images. The approach is not limited to ultrasound data and was experimentally applied to $3 D-C T$ and MRI data as well.
\end{abstract}

\section{Introduction}

Development of Live-3D ultrasound imaging has increased the acquisition speed for echocardiography. Compared to rotational or freehand 3D ultrasound imaging motion artifacts are reduced, which could result in more accurate volume analysis. To benefit from this noninvasive realtime imaging modality and to perform a more accurate analysis of the heart function efficient postprocessing of the data is necessary. The first step for volumetric and functional analysis is the delineation of the left and/or right ventricles endocardium in the complete data set. Common two-dimensional segmentation methods are very time consuming and often result in inconsistent segmentations. Automatic intensity based segmentation methods fail due to the enormous amount of noise and speckle in ultrasound images.

The use of deformable models can improve the (semi-) automatic segmentation of noisy data. Different methods were presented $[1,2,3]$. Nevertheless the application of such models is still limited. The parametrization always strongly depends on the quality of the acquired data and the subject under examination. In most cases model parameters need to be modified slightly from one data set to another. Because there is no general rule for model parametrization, parameters can only be found experimentally, which makes it a time consuming task again and often can only be performed by experts. This is not suited for a tool usable in clinical routine.

A very promising approach for segmentation of the left ventricle in 3D time series was presented by Gerard et al. [4]. Their described method consists of several steps starting with a rough semi-automatic segmentation of the first 3D data set by interactively defining 4 anatomical landmarks. The approach still has the disadvantage of difficult model parametrization. Furthermore, necessary user interaction in the possible event of failure of the semiautomatic segmentation procedure is unclear. Frangi et al. concluded in [5] this aspect cannot be neglected and "the desirable end goal, the full automation, is very difficult and user-interaction is almost always unavoidable".

As Suri concluded in [6] one of the main demands in cardiac imaging is to build a fast robust, computationally inexpensive cardiac analysis tool.

The major goal of this work was to develop such an easy to use semi-automatic segmentation tool for Live3D echocardiographic images. User interaction should be reduced to a minimum while still allowing for correction and modification of the underlying automatic deformable model segmentation.

\section{Methods}

Fig. 1 shows the workflow of the new segmentation tool. After loading a new data set the user selects one time frame and defines attractors in regions where he wants the segmentation to pass through and where the boundaries of the structure of interest are not clearly visible. Then an initial surface model enclosing all defined attractors is computed. In the next step the initial model is deformed according to image forces, model forces and until it 
passes through all defined attractors. The user continously gets an update of the current status of segmentation in two-dimensional multiplanar orthogonal views as well as in a three-dimensional surface model view. While the segmentation continues the user can add more attractors to influence the model in regions where the deformation stopped too early or correct the surface model in cases of over-segmentation until he is satisfied with the result. Then the next time frame can be selected. The previously defined attractors can be used for segmentation of this next frame. They can interactively be moved to the new boundaries and a new surface model is fitted through the attractors and to the image data.

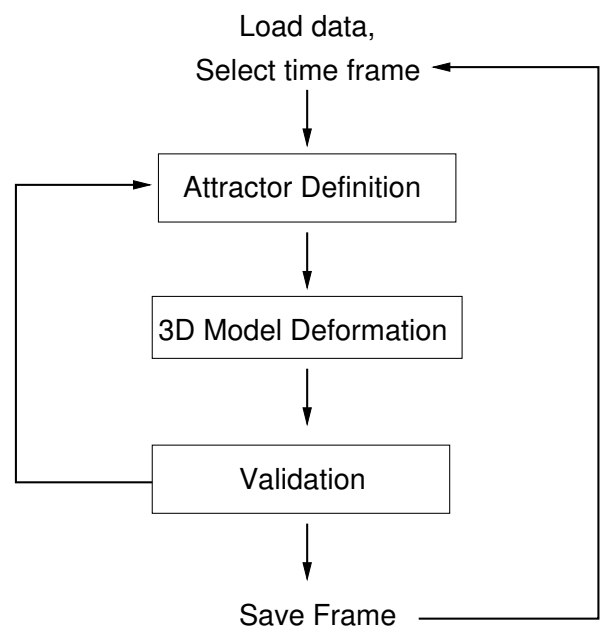

Figure 1. Workflow of the volume segmentation tool.

\subsection{Deformable Model}

Similar to [4] the implemented deformable model is based on a 2-simplex mesh representation, which is a discrete closed non-parametric surface representation in $R^{3}$, where each point is connected to exactly three neighbors [3]. A simplex mesh $M$ consists of a vertex set $\left\{p_{i}\right\}$. Simplex meshes provide good capabilities to control the local geometry of the surface and allow to efficiently compute discrete geometric properties.

\subsubsection{Deformation scheme}

In an iterative scheme each vertex $p_{i}$ is moved according to the discrete approximation of the Newtonian law of motion:

$$
p_{i+1}=p_{i}+\alpha F_{i n t}+\beta F_{\text {ext }}+\iota F_{\text {attr }}
$$

The internal force $F_{\text {int }}$ influences the smoothness of the surface and follows the approach in [3]. The external force $F_{\text {ext }}$ is computed using a Gaussian gradient filter after initial anisotropic diffusion filtering of the input image [7]. $\quad F_{a t t r}$ is the newly introduced attraction force component constraining the mesh deformation. Its computation is based on a set of points (attractors) $\left\{a_{i}\right\}$ and their Euclidean distance to the mesh. To guarantee stability of the mesh deformation the force components are scaled by $\alpha, \beta$ and $\iota$.

\subsubsection{Topology adaption}

To cope with complex surface geometries it is necessary to reconfigure the topology of the surface model. Therefore several topology adaption operations were implemented. The most important operation is the insertion of new points in places of high curvature enabling the surface mesh to grow into small cavities. This operation is performed automatically during model development where the average curvature of a surface cell gets too high. New points are inserted in this cell and the cell is split. On the other hand cells that do not satisfy a minimum curvature criteria are joined by deleting shared edges. Other properties than average curvature can be chosen as criteria for topology modifications. Currently, a second feature is implemented taking the size of cells into account, which is useful for generating meshes with regular cell sizes.

\subsection{User interaction}

Prior experiences with interactive two-dimensional segmentation tools [8] showed that new interaction schemes should carefully be developed. They need to be easy to understand and intuitive to handle. The radiologists should easily remember how to use such a tool even if it is used rarely. Several interaction schemes for definition of the attraction forces were tested. They were integrated in the first version of the new tool enabling us to identify the interaction method preferred by our clinicians.

The first and simplest interaction method is based on the definition of points. Points can be added, moved and deleted in the two-dimensional multiplanar views. The second scheme lets the user draw opened or closed contours, correct them and delete them. The attractor forces are extracted from these contours by subsampling points from the contours. The last interaction scheme extends the second approach by integrating a whole palette of two-dimensional segmentation tools, e.g. an interactive region grower, a thresholding tool and a polygon tool. The latter approach is especially interesting for another possible application of the new tool, the segmentation of 3D-CT heart data. Here the interactive region grower can be used to delineate a few orthogonal slices of the LV endocardium. Then the workflow can be applied as presented here except for a different smoothing and image 


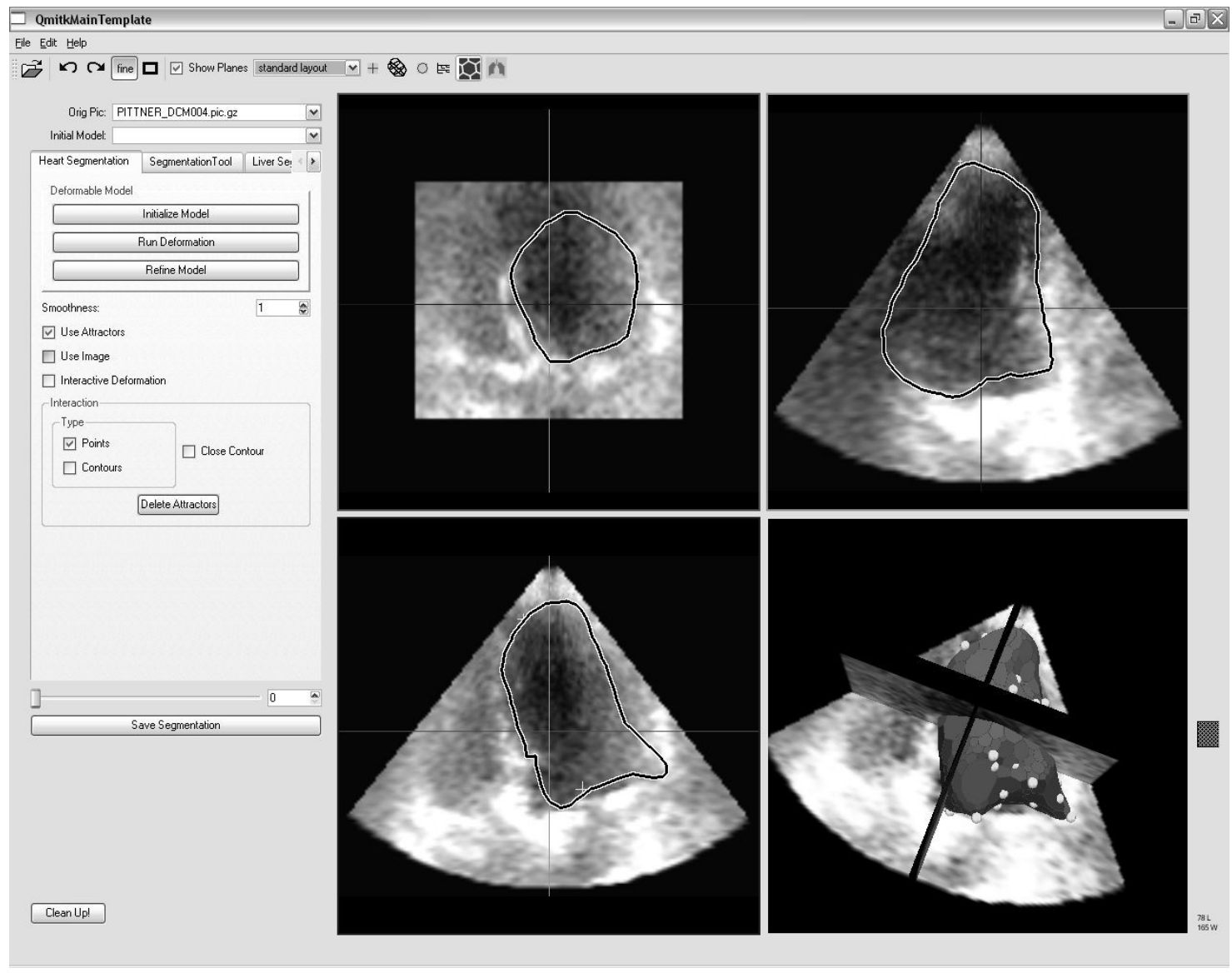

Figure 2. User interface containing the three multiplanar two-dimensional views and a three-dimensional preview window (bottom right). The latter shows the result of the segmentation process, the surface model of the left ventricle, together with the user-defined attractors (small light points) overlaid with cross-sections of the original echo data.

force computation of the input data.

\subsection{Implementation}

The developed application was implemented using the Medical Imaging \& Interaction Toolkit (MITK) [9]. The basic deformable model and topology adaption algorithms were integrated into the open-source "Insight Segmentation \& Registration Toolkit" (ITK) [10] and are available via free download since version 1.8.

\section{Results}

The new application was used for delineation of left (LV) and right ventricles (RV). Looking towards clinical application the biggest advantage is the simplified use. No parameter adjustment is necessary. After loading the data the user can directly start by drawing the attractors. Then the initial model is fitted to the attractor data and the image data. Fig. 2 shows the screenshot of the user interface.
Because the attractor forces are implemented as hard constraints, it is always guaranteed that the resulting segmentation passes exactly through the user defined points. This has the effect that a larger part of the surface model is attracted by these forces, as mesh points near an attracted point always follow their moving neighbors due to the minimization of the internal energy of a simplex mesh. When such points get closer to high image gradient forces they get attracted by these image forces as well. This positive attractor force influence stabilizes and speeds up the deformation process.

\subsection{Surface Mesh Topology}

The final surface mesh of a delineated left ventricle consists of approximately 250-400 surface points (average). Due to the more complex geometry of the right ventricle the final surface meshes consist of 450-800 points. 


\subsection{User Interaction}

The different approaches for attractor definition were tested. The simplest one, the point definition scheme was also the most successful. For delineation of a left ventricle around 15 to 25 points needed to be set until the user was satisfied with the result. For segmentation of right ventricles more points $(30-40)$ were needed. This can be explained by the higher geometric complexity of the right ventricle.

\subsection{Time}

First evaluation showed the new tool can reduce the time needed for delineation of Live-3D echocardiographic data. The delineation of the LV in one time frame of a data set approximately takes 2 minutes. For analysis of the ejection fraction $(\mathrm{EF})$ the end diastolic and end systolic time frame need to be delineated only, which makes a total time of just 4 minutes. Following the delineation of a complete Live-3D data set with 15 time frames takes about half an hour. Compared to manual segmentation, where the segmentation of one time frame approx. takes 15 minutes per ventricle the total time needed for delineation of both LV and RV could significantly be reduced using the here presented tool.

\section{Discussion and conclusions}

The major goal, developing a robust semi-automatic segmentation tool was achieved. It could be shown that the presented tool is suited for the segmentation of Live3D echocardiographic images.

The chosen approach furthermore combines the ability of deformable models to automatically detect object boundaries with interactive controls enabling the user to influence the models development and ensuring accuracy of the results. By giving direct feedback, i.e. segmentation preview, the user can always see how the three-dimensional surface model develops and deforms. The parametrization of the deformable model is hidden from the user who can use the tool straightforward, without knowing anything about deformable model parametrization.

Current work deals with extending the approach for the segmentation of $3 \mathrm{D}+\mathrm{t}$ data. Successive time frames of the original data are registered using optical flow. From registration a deformation field is obtained, which can be used to automatically shift the interactively defined attractors. By successfully applying this method the total amount of time needed for the segmentation of ventricles in the complete $3 \mathrm{D}+\mathrm{t}$ data set could be reduced to less than ten minutes.

\section{Acknowledgements}

This work is supported by the Deutsche Forschungsgemeinschaft within the Special Research Area 414 "Information Technology in Medicine - Computer and Sensor Supported Surgery".

\section{References}

[1] Kass M, Witkin A, Terzopoulos D. Snakes: active contour models. International Journal of Computer Vision 1988; 1(4):321-331.

[2] Neuenschwander W, Fua P, Szekely G, Kubler O. Deformable velcro surfaces. In Lemke $\mathrm{H}$, Vannier M, Inamura K, Farman A (eds.), Proceedings of the International Conference on Computer Vision. Cambridge, $1995 ; 828-833$.

[3] Delingette H. General object reconstruction based on simplex meshes. International Journal of Computer Vision 1999;32(2):111-146.

[4] Gerard O, Billon A, Rouet JM, Jacob M, Fradkin M, Allouche C. Efficient model-based quantification of left ventricular function in 3-d echocardiography. IEEE Transactions on Medical Imaging September 2002; 21(9):1059-1068.

[5] Frangi AF, Niessen WJ, Viergever MA. Three-dimensional modeling for functional analysis of cardiac images: A review. IEEE Transactions on Medical Imaging January 2001;20(1):2-25.

[6] Suri JS. Computer vision, pattern recognition and image processing in left ventricle segmentation: The last 50 years. Pattern Analysis Applications 2000;3(3):209-242.

[7] Montagnat J, Sermesant M, Delingette H, Malandain G, Ayache N. Anisotropic filtering for model-based segmentation of $4 \mathrm{~d}$ cylindrical echocardiographic images. Pattern Recognition Letters 2003;24(4-5):815-828.

[8] Kunert T, Cardenas CE, Diehl S, Düber C, Meinzer HP. Problems of interactive segmentation. Biomedizinische Technik 2002;47(Suppl 1 Pt 2):933-935.

[9] Wolf I, Vetter M, Wegner I, Boettger T, Nolden M. The Medical Imaging Interaction Toolkit (MITK) - a toolkit facilitating the creation of interactive software by extending VTK and ITK. In Medical Imaging: Image Processing, volume 5367. SPIE, 2004; 0-0.

[10] Ibanez L, Schroeder W, Ng L, Cates J. The ITK Software Guide. Insight Software Consortium, 2003. URL http: //www.itk.org/ItkSoftwareGuide.pdf.

Address for correspondence:

Thomas Boettger

German Cancer Research Center

Div. Medical and Biological Informatics B010

Im Neuenheimer Feld 280

69120 Heidelberg, Germany

t.boettger@dkfz.de 